\title{
Filigrane
}

Écoutes psychanalytiques

\section{De la transidentité du patient au vacillement identitaire du clinicien}

\section{Nicolas Evzonas}

Volume 28, numéro 2, 2019

Identités $^{2}$. Qui suis-je ? Deuxième partie

URI : https://id.erudit.org/iderudit/1069692ar

DOI : https://doi.org/10.7202/1069692ar

Aller au sommaire du numéro

\section{Éditeur(s)}

Santé mentale et société

ISSN

1192-1412 (imprimé)

1911-4656 (numérique)

Découvrir la revue

Citer cet article

Evzonas, N. (2019). De la transidentité du patient au vacillement identitaire du clinicien. Filigrane, 28(2), 11-29. https://doi.org/10.7202/1069692ar

\section{Résumé de l'article}

L'auteur de cet article se propose de déplacer le curseur habituellement mis sur la conflictualité des patient/e/s vers la subjectivité du/de la clinicien/ne. Il interroge ainsi son identité de psychothérapeute orienté par la métapsychologie et ses défis contre-transférentiels dans le cadre de son travail en institution avec des adultes transgenres. En narrant les vicissitudes de la psychothérapie psychanalytique de David, patient composite pensé à partir des cures de plusieurs sujets aux identifications de genre atypiques, il esquisse les divers moyens de métaréflexion clinique qu'il a envisagés afin d'ajuster sa position, systématiquement compromise par les problématiques identitaires du patient, ainsi que par ses propres préjugés et réactions inconscientes. Il décrit en outre comment le contexte contemporain des transidentités marqué par l'activisme et la rectitude politique est susceptible d'entraver la vocation analytique consubstantielle à l'exploration de l'inconscient par définition a-normatif et politiquement incorrect.
Ce document est protégé par la loi sur le droit d'auteur. L'utilisation des services d’Érudit (y compris la reproduction) est assujettie à sa politique d'utilisation que vous pouvez consulter en ligne.

https://apropos.erudit.org/fr/usagers/politique-dutilisation/ 


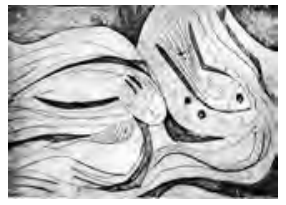

\title{
De la transidentité du patient au vacillement identitaire du clinicien
}

\author{
Nicolas Evzonas
}

\begin{abstract}
Résumé: L’auteur de cet article se propose de déplacer le curseur habituellement mis sur la conflictualité des patient/e/s vers la subjectivité du/de la clinicien/ne. Il interroge ainsi son identité de psychothérapeute orienté par la métapsychologie et ses défis contre-transférentiels dans le cadre de son travail en institution avec des adultes transgenres. En narrant les vicissitudes de la psychothérapie psychanalytique de David, patient composite pensé à partir des cures de plusieurs sujets aux identifications de genre atypiques, il esquisse les divers moyens de métaréflexion clinique qu'il a envisagés afin d'ajuster sa position, systématiquement compromise par les problématiques identitaires du patient, ainsi que par ses propres préjugés et réactions inconscientes. Il décrit en outre comment le contexte contemporain des transidentités marqué par l'activisme et la rectitude politique est susceptible d'entraver la vocation analytique consubstantielle à l'exploration de l'inconscient par définition a-normatif et politiquement incorrect.
\end{abstract}

Mots clés: transgenre; contretransfert; homosexualité; supervision; intervision ; écriture.

\begin{abstract}
The author of this article proposes to shift the focus usually placed on the conflict of patients toward the subjectivity of the clinician. He thus questions his identity as a metapsychologically oriented psychotherapist and his counter-transferential challenges in working with transgender adults in an institutional setting. By narrating the vicissitudes of the psychoanalytical psychotherapy of David, a composite case stemming from the treatment of several individuals with atypical gender identifications, the author describes the various means of clinical metareflection that he considered in order to adjust his stance, which was systematically compromised by the patient's issues as well as his own prejudices and unconscious reactions. He further describes how the contemporary context of trans identities shaped by activism and political correctness is likely to hamper the analytical vocation to explore the unconscious, which is intrinsically a-normative and politically incorrect.
\end{abstract}

Keywords: transgender; counter-transference; homosexuality; supervision; intervision; writing. 


\section{L'inversion de la question trans'}

/ oici seize ans, Éric Fassin (2003) a inversé la «question homosexuelle» en se penchant sur la façon dont l'homosexualité (ré) interrogeait notre savoir disciplinaire plutôt que sur la manière dont ce dernier problématisait l'homosexualité. Dans le cadre de cet article, nous nous proposons de suivre un chemin analogue concernant les transidentités. En conséquence, il ne s'agira pas d'envisager celles-ci comme objet d'analyse mais, inversement, de mettre la psychanalyse à l'épreuve du discours trans'. Plus précisément, nous réfléchirons sur les effets transférentiels que les sujets transgenres sont susceptibles d'induire chez le/la clinicien/ne orienté/e par la métapsychologie. On pourrait poser la question en ces termes: la théorisation psychanalytique sur lesdites «dysphories de genre» ne résulterait-elle pas d'une résistance contretransférentielle à la demande des patient/e/s trans'?

Le survol de la littérature psychanalytique francophone et internationale permet de déceler un contretransfert équivoque vis-à-vis des individus aux identifications de genre atypiques. Cette posture est dénoncée comme normative, voire transphobe aussi bien par les activistes ou chercheur/se/s trans' (Califia, 2003; Espineira, 2015; Preciado, 2008) que par certain/e/s psychanalystes contemporain/e/s (Gozlan, 2008, 2018; Saketopoulou, 2014; Ayouch, 2015; Barkai, 2017; Hansbury, 2017). Pour notre part, nous avons choisi de nous extraire de la position sans doute plus confortable de critique de la posture des autres analystes, préférant nous focaliser sur nos propres difficultés et vacillements identitaires dans le cadre de notre travail en institution avec des adultes transgenres. Si «le compte rendu d'un cas clinique expose l'analyste, sa théorie et sa pratique bien plus que ne peut le faire aucun texte théorique» (Aulagnier, 1984, p. 13), centrer ses propos sur son contretransfert n'impliquera-t-il pas une exposition maximale? Nous avons néanmoins pris ce risque car nous avons voulu marquer une distance visà-vis du/de la clinicien/ne-héros/ïne, à l'abri de tout conflit, replié/e sur son savoir arrogant et l'infaillibilité de sa posture. Nous espérons ainsi contribuer à renforcer la réflexivité critique des analystes qui ont longtemps pathologisé les transidentités jusqu'à frôler à l'occasion la maltraitance clinique et théorique des sujets concernés.

Nos propos dans cette contribution seront étayés par notre expérience avec David, âgé d'une trentaine d'années. Il s'agit en vérité d'un «cas composite» (Gabbard, 2000), formé à partir des plusieurs sujets transgenres suivi/e/s par nous en institution dans le cadre de psychothérapies psychanalytiques en face-à-face. Ce choix nous a été primordialement dicté par 
le souci de confidentialité et secondairement par les problématiques trans' qui mettent en exergue l'hybridation et le métissage. Nous faisons nôtres ici les propos du psychanalyste américain Griffin Hansbury (2011), lorsqu'il explique sa décision de créer un "analysant composite " plutôt que de narrer une série fragmentée de vignettes cliniques:

Si celui-ci n'est pas entièrement réel, il possède une vraisemblance qui est assez bonne. Cet acte de rassembler et de tisser ensemble des parties disparates en un tout utilisable ressemble à ce que font les trans' dans le processus de construction de leur corps et leur être, à la recherche d'une forme apparemment sans couture - bien que ce soit aux coutures secrètes, dans leurs déchirures et leurs lacunes, que se produit le frisson de la friction. J'espère que cet article sera rempli de lacunes de cet ordre. (p. 211; nous traduisons).

\section{L'obstacle du savoir pré-transférentiel ou la communication paradoxale}

David, assigné «fille» à sa naissance, a souhaité entamer un suivi psychothérapique en vue d'une transition hormono-chirurgicale vers le genre masculin. Nous avons réalisé progressivement que ce qui l'avait incité au premier chef à entreprendre cette démarche était son angoisse de ressembler aux femmes violentes de sa famille. Lors d'une séance, il a formulé sans ambages cette crainte: «Ma hantise, c'est de devenir comme elles». David disait souvent que nous étions "doux, calme et posé» en nous situant vraisemblablement aux antipodes des personnes agressives qu'il détestait. Il réitérait en outre à quel point il était satisfait de nos séances car il se sentait enfin libre de pouvoir exprimer ce qu'il avait étouffé pendant toute sa vie «pour ne pas perdre l'affection de son entourage et se retrouver seul». La crainte du désamour et de l'isolement aurait été le motif qui l'avait poussé à atermoyer la décision de concrétiser sa transition vers le genre masculin en choisissant la solution plus « anodine» du saphisme.

Dans les premiers mois de sa cure, le récit de David a laissé transparaître une idéalisation extrême du modèle traditionnel de la famille hétérosexuelle, ce qui nous a incité à penser que sa transidentité camouflait une homosexualité inassumée. Malgré le fait qu'il abordait son lesbianisme comme un pis-aller, nous nous obstinions à entendre une défense contre l'homosexualité. Dans le cadre de l'écriture sur ce cas, qui a généré chez nous les effets d'une supervision, nous nous sommes surpris à découvrir 
que cette piste nous avait été insidieusement dictée par la réduction des transidentités à l'homosexualité refoulée qui a prévalu dans une certaine littérature psychanalytique dogmatiquement attachée à l'approche freudienne du cas Schreber (Castel, 2003; Freud, 1911). Lorsque nous avons pu nous défaire de ce vieux poncif métapsychologique grâce à un travail d'intervision entrepris avec quelques pairs, nous avons su entendre les paroles de David et nous confronter à l'hypothèse inverse de l'homosexualité comme défense contre la transidentité. «Mon lesbianisme était un stratagème pour atténuer ma dysphorie de genre, en quête d'un lieu où je pourrais avoir un comportement plus masculin ou, au moins, être un autre genre de femme.» Comment ne pas avoir su écouter auparavant ce qui était pourtant si clairement formulé?

Michel Neyraut (1974) a évoqué l'existence d'un contretransfert antérieur à la situation analytique, indissociable du développement historique de la pensée psychanalytique et des normes socioculturelles qui encadrent ce dernier. Autrement dit, il existerait un savoir pré-(contre) transférentiel, idéologiquement chargé, capable d'orienter mais aussi de désorienter l'écoute de l'analyste. Jean Laplanche (1987, p. 12) a parlé pour sa part d'un «terrorisme des concepts implicites» qui peut s'imposer sous le nom de retour à la clinique. Avant lui, Lacan (1969-1970) avait averti que les «signifiants-maîtres» étaient susceptibles de constituer un piège pour le psychanalyste. Piera Aulagnier (1984) a résumé quant à elle la difficulté du «métier impossible» de l'analyste comme l'exigence d'accomplir deux fonctions paradoxales dans l'espace de la cure: celle d'un «sujet supposé savoir» et celle d'un «sujet supposé ignorant». Pour notre part, nous nous sommes rendu compte de la difficulté, dans ce cas précis, de concilier la métapsychologie avec l'attention également flottante censée mettre en suspens le déjàsu pour laisser émerger l'inconnu. La conception des transidentités comme une homosexualité défensive était, semble-t-il, tellement ancrée dans notre formation qu'elle nous rendait sourd aux énoncés de David.

On rappellera ici ce que Laplanche (2003, p. 163) écrit à propos de «l'impensé» de la catégorie du genre dans l'analyse freudienne du président Schreber:

Toute la dialectique du «Moi (un garçon/je l'aime, lui [un garçon]» se centre sur la seconde partie de la phrase sans jamais mettre en question ce que veut dire «moi un garçon». Problématique, pourtant, qui est directement celle de Schreber, et que beaucoup d'analystes, à juste titre, ont 
rapproché de celle du transsexualisme. En psychanalyse, l'immense majorité, voire la totalité des observations pose de façon irréfléchie au départ: «il s'agit d'un homme de trente ans; ou d'une femme de vingt-cinq ans, etc.» Le genre serait-il vraiment a-conflictuel au point d'être un impensé de départ? Aurait-il pour ainsi dire expulsé le conflictuel en dehors de lui sous la forme du sexual?

Dans cette perspective, l'assimilation des transidentités à une homosexualité refoulée pourrait être interprétée comme une construction défensive visant à dénier l'inquiétante étrangeté du genre. Et toute théorie constituerait une conjuration de l'angoisse née d'une énigme traumatique.

En dépit de sa pertinence métapsychologique, cette hypothèse risque de maintenir dans un état de refoulement l'inscription historique des transitions hormono-chirurgicales de genre devenues possibles à partir de la deuxième moitié du $\mathrm{xx}^{\mathrm{e}}$ siècle. Les analyses du philosophe foucaldien Arnold I. Davidson rappellent à cet égard que les «impensés conceptuels» constituent souvent des «impensés historiques». En ce qui concerne les transidentités, Davidson soutient que:

avant le milieu du XIX ${ }^{e}$ siècle, les personnes d'un sexe anatomique déterminé ne pouvaient être considérées comme appartenant psychologiquement au sexe opposé, puisque, à l'époque, le sexe anatomique subsumait l'identité de genre d'une personne. [...] Lesdites opérations de réattribution sexuelle n'étaient pas seulement technologiquement impossibles dans les siècles précédents; elles étaient aussi impossibles sur le plan conceptuel. (Davidson, 1987, p. 22; nous traduisons)

Selon cette logique soucieuse de l'historicisation du savoir, la psychanalyse à ses débuts n'aurait pu appréhender les transidentités qu'en référence aux catégories conceptuelles disponibles en son temps. Par conséquent, l'idée d'une homosexualité détournée serait cohérente avec la subjectivité d'une époque qui assimilait le désir homoérotique à l'appartenance à l'autre genre normativement défini. Quand on se forme à la métapsychologie, c'est tout un héritage culturel qu'on absorbe souvent à son insu.

La suite de la cure de David nous a réservé un revirement inattendu. Évoquant la reprise de contact avec sa mère, le patient a décrit la détresse induite par la réaction enragée de cette dernière envers son projet de subir une chirurgie de réaffectation génitale: "Je ne comprends pas ce rejet; 
pourtant je vais devenir un homme; je vais enfin me normaliser; je ne serai plus la gouine que ma mère a toujours détestée. Lorsque j'ai fait un coming out forcé pendant mon adolescence, elle me menaçait constamment de me mettre à la porte parce que j'étais une abomination pour elle. Finalement, je vais rester à tout jamais une abomination.» Cette parole, qui a réémergé ultérieurement au sein des associations sur son père homosexuel et, de ce fait, disqualifié et haï par sa mère, suggérait une homophobie internalisée en arrière-fond de l'identification transgenre. Pendant plusieurs séances, alors que ce thème revenait avec force, nous étions incapable de penser ou de formuler le moindre questionnement. Quelque chose nous empêchait de suivre la processualité de la cure. Ce n'est que dans l'après-coup de la lecture de nos notes que nous avons soupçonné que cette paralysie était sans doute liée à notre difficulté de nous affranchir d'un discours dit progressiste qui récusait comme archaïque et rétrograde l'association des transidentités à l'homosexualité déniée ${ }^{1}$, lorsqu'il n'interdisait pas toute articulation entre sexualité et identité de genre ${ }^{2}$. Le fantôme du savoir pré-transférentiel ne faisait-il pas sa réapparition sous une forme inversée?

Les choses se sont avérées bien plus complexes. À force d'écouter David, l'idée d'une communication paradoxale entre lui et sa mère s'installait progressivement chez nous: celle-ci ne lui aurait-elle pas inculqué d'une part son rejet haineux de l'homosexualité et, d'autre part, son désir ambivalent vis-à-vis d'un époux qu'elle avait d'emblée su homosexuel? Ne serait-on pas ici au plus près de ce que Searles (1957), Aulagnier (1984), Roussillon (1991) et bien d'autres psychanalystes ont décrit, à la suite de Bateson et ses collaborateurs, comme une situation à «double lien» ou «double entrave» (double bind)? Selon cette optique, David aurait introjecté les messages antinomiques que sa mère lui aurait transmis et notre paralysie en séance constituerait la traduction contretransférentielle d'une atteinte de la pensée induite par lesdites injonctions paradoxales.

Cette prise de conscience n'a pas été suffisante pour rétablir notre posture et relancer notre attention également flottante. Il a fallu une supervision grâce à laquelle nous avons pu analyser les effets de la communication paradoxale, cependant reproduite méta-transférentiellement au sein de la relation avec notre superviseur. Par ailleurs, une intervision, sans doute grâce à la latéralisation et à la pluralisation du (méta) transfert, nous a permis de concilier ce que Hansbury (2011, p. 211) appelle avec humour le savoir du «Père freudien moderne» (la métapsychologie classique) et celui de la «Mère féministe post-moderne» (le discours progressiste sur le genre). 


\section{L'identification à l'agresseur ou le remède du nouveau savoir}

La cure de David a généré chez nous un autre effet inattendu. La répugnance exprimée par le patient vis-à-vis de son corps «femelle», qui supplantait tous ses éloges de la virilité, s'est avérée déstabilisante pour nous. L'horreur de la menstruation, le dégoût de ses seins, la haine de son utérus et le vertige associé à l'idée de la grossesse nous ramenaient inexorablement aux conclusions de certain/e/s auteur/e/s qui tenaient des propos violents à l'égard de leurs patient/e/s transgenres ${ }^{3}$. L'aphorisme de Colette Chiland (2011, p. 163) «être un homme, c'est échapper à l'horreur d'être une femme», que nous considérions comme radicalement réducteur et disqualifiant, se concrétisait avec clarté à travers les énoncés de David. Notre ébranlement subjectif fut tel qu’il nous éloigna aussi bien de la neutralité que de la bienveillance, par souci d'invalider cette vérité émergente et d'esquiver ainsi notre identification à l'agresseur. «Ma hantise, c'est de devenir comme elles »: l'angoisse de David de s'assimiler aux femmes violentes de son entourage concordait avec notre crainte de nous assimiler aux auteur/ $\mathrm{e} / \mathrm{s}$ maltraitant/e/s, comme si nous avions incorporé la problématique du patient. Lui en voudrions-nous parce qu'il nous aurait atteint viscéralement? Qui était le plus agissant au sein de notre dyade?

Nous nous sommes entendu un jour lui poser cette question surprenante: "Dans un monde idéal vous auriez choisi la case "Monsieur", la case "Madame", la case "Autre" ou encore une autre case que vous auriez inventée?» Sa réponse: "Je choisirais "Monsieur" car par notre éducation on est habitué à choisir soit l'un soit l'autre; si ce n'est pas "Madame", ça doit obligatoirement être "Monsieur"». Nous avons poursuivi ou plutôt nous l'avons poursuivi: «C'est pour ça que j'ai dit dans un monde idéal. Car vous choisissez "Monsieur" comme par défaut ». À force de vouloir démentir la théorie précitée qui nous déplaisait («être un homme, c'est échapper à l'horreur d'être une femme»), nous avions adopté un style comminatoire à l'exemple des clinicien/ne/s que nous critiquions, notre attitude équivalant à un refus de la parole du patient au lieu d'une incitation à faire advenir cette parole. Piégé dans nos contradictions, nous avons méconnu l'avertissement d'un Ferenczi (1928, p. 61) particulièrement sensible aux phénomènes du contretransfert: «Être économe d'interprétations, en général, ne rien dire de superflu, est une des règles les plus importantes de l'analyse; le fanatisme de l'interprétation fait partie des maladies d'enfance de l'analyste».

Outre l'effet précieux de la supervision et de l'intervision, notre familiarisation avec un certain nombre de nouvelles lectures dédiées au genre nous 
a aidé à atténuer le conflit entre nos postulats théoriques et nos constatations cliniques. Citons à titre d'exemple un extrait de Judith Butler (2009, p. 13) qui met précisément en garde contre des observations qui se rapprochent des nôtres: «Les désirs positifs de changer de sexe ne sont pas toujours ancrés dans la répudiation du genre précédent, et le cadre binaire et la logique de non-contradiction ne s'appliquent pas dans ce domaine [...]. Ce que veut une personne ne doit pas toujours être compris en termes de ce qu'elle rejette ou souhaite mettre de côté».

On remarquera de prime abord que Butler admet elle aussi l'existence d'une tendance à s'approprier un genre en passant par le reniement d'un autre, sauf qu'elle s'élève contre la généralisation de ce postulat en loi absolue. Nous partageons cette réserve car ce que l'on peut relever dans une cure peut être infirmé par une autre, dans la mesure où chaque sujet est irréductiblement singulier. Julia Kristeva (2003) a très justement décrit la psychanalyse comme une "poétique de la singularité». Cela dit, nombreux/euses furent les analystes d'orientation freudienne à ranger tou/te/s les trans' dans la catégorie des états limites et les clinicien/ne/s lacanien/ne/s à les indexer indistinctement à la psychose ${ }^{4}$. Une telle systématisation n'est-elle pas antianalytique au plus haut point?

Grâce aux travaux de Laqueur (1992), de Butler (2009), de Laplanche (2006) et d'autres, nous avons commencé à «débloquer» notre écoute et à nous sensibiliser à l'implication des enjeux culturels dans «la fabrication du corps sexué qui ne se cantonne [rait] pas à une simple grammaire du sujet» (Laufer, 2010). Nos nouvelles lectures, en combinaison avec l'accroissement de notre expérience clinique, nous ont permis de complexifier la logique intrapsychique de l'identité de genre en établissant une corrélation entre le renoncement au «sexe génétique» et l'angoisse de «performer» un idéal de genre socialement construit qui se situerait hors de portée du sujet. Cette tension pourrait aboutir, sous la pression d'une binarité culturellement instituée, transmise et compromise par l'inconscient parental, au choix du genre opposé. Penchons-nous sur une nouvelle séquence clinique de la cure de David pour illustrer nos propos.

Selon notre patient, sa mère avait cherché à lui inculquer sa propre image de la féminité en lui imposant des postures de danseuse et des manières de table de jeune fille de bonne famille. Cet endoctrinement se serait accompli notamment par le biais d'ouvrages prescriptifs véhiculant une approche clivée, binaire et hétéronormative de la relation entre les genres. David a insisté sur son obstination à déconstruire ces consignes tyranniques en adoptant 
des contre-attitudes masculines. Il a relaté en outre qu'il cherchait désespérément à repérer dans le miroir la créature sublime esquissée dans les «bibles de genre» fournies par sa mère. Nonobstant, en lieu et place de cette beauté redoutablement inaccessible, il était confronté, à l'image de fille imparfaite que ses camarades de collège lui renvoyaient sans pitié, à grand renfort de clichés relatifs au maquillage, à l'épilation et aux robes moulantes. Dans cette optique, l'identification transgenre aurait constitué une tentative de déprise d'un idéal de genre contraignant, façonné autant par les désirs maternels que par les normes socioculturelles médiatisées par la mère et refiltrées par le groupe de pairs adolescents. David a expliqué qu'il aurait pu choisir de devenir une «fille potable» en se maquillant, mais que, faute de pouvoir être «ravissante», conformément au modèle décrit dans les ouvrages sus-cités (mentionnons le titre évocateur de l'un de ces livres: Comment être une fille parfaite), il s'était fait couper les cheveux très courts grâce à quoi il avait enfin aperçu dans la glace un reflet séduisant: celui d'un beau gosse.

Aujourd'hui, nous sommes enclin à penser que plus les injonctions de genre sont rigides au sein d'un contexte familial et social donné, plus il est ardu pour l'enfant de s'y plier et plus il/elle risque de développer des contreattitudes. Selon cette logique, le choix de la transition constituerait un destin possible de la difficulté que nous rencontrons tou/te/s lorsqu'il s'agit de nous situer dans le dimorphisme masculin/féminin imposé par notre culture.

\section{Confusion des langues}

Si la pluralisation de nos lectures a contribué à un élargissement de notre écoute et à une meilleure maitrise de notre contretransfert, nous étions par ailleurs confronté à un autre obstacle: l'utilisation du pronom masculin pour s'adresser à David ne nous venait pas naturellement, si bien que nous alternions involontairement entre le féminin correspondant au genre assigné à sa naissance et le masculin conforme à son genre vécu ${ }^{5}$. Pourquoi nous écartions-nous de notre intention d'adhérer au langage du patient puisque nous savions par expérience que le «mégenrement» induit une violence inouïe chez les sujets trans'? Dans le cadre de notre analyse, nous avons pris conscience que, malgré notre positionnement supposément progressiste en matière de genre et notre imprégnation par le regard analytique qui ne relève pas de la «vision tautologique» - ce que je vois est ce que je vois (Didi-Huberman, 1992) -, nous avions du mal à nous affranchir de la congruence sexe/genre imprimée sur nous par les normes dominantes non seulement socioculturelles, mais aussi, et surtout, métapsychologiques. 
À un niveau plus profond, nous avons traversé l'expérience d'un vacillement identitaire, contre lequel nous nous sommes défendu en attribuant à David une rigidité de genre.

Paul Denis comprend ce phénomène comme un clivage contretransférentiel:

Dans la plupart des cas, un trouble de l'identité de genre chez un patient engendre chez l'analyste une sorte de flottement, de désorganisation, tant nous sommes organisés autour de la différence des sexes et des générations, tant nous sommes habitués à nous adresser à quelqu'un en fonction du fait qu'il est une femme ou un homme. Cette gêne peut être considérable et induire une forme de clivage contretransférentiel particulier sur le modèle: "Je sais bien, mais quand même»; je sais que ce patient est une femme, mais quand-même elle est un homme. (2013, p. 767)

Leslie Lothstein (1979, p. 73) écrit quant à lui que «cette confusion se manifeste souvent par un état d'ennui, de fatigue ou d'hostilité active à l'égard un patient» lequel aboutit à deux contre-attitudes: «le déni et le détachement ou la suridentification et l'acceptation".

Pour notre part, afin de dépasser ce clivage, nous avons décidé, au lieu d'adopter le masque du thérapeute marmoréen, abstentionniste et imbu de lui-même, de faire nôtres les consignes de Ferenczi (1933, p. 128), qui a dénoncé l'hypocrisie professionnelle de ses collègues en prônant la sincérité, seule qualité apte à instaurer la confiance entre analyste et analysant:

En réalité, il se peut que certains traits, externes et internes du patient nous soient difficilement supportables. Ou encore, nous sentons que la séance d'analyse apporte une perturbation désagréable à une préoccupation personnelle et intime. Là aussi je ne vois pas d'autre moyen que de prendre conscience de notre propre trouble et d'en parler avec le patient, de l'admettre, non seulement en tant que possibilité mais aussi en tant que fait réel.

En conséquence, nous avons prévenu notre patient de la fluctuation lexicale, qui risquait de se reproduire en dépit de notre acceptation de sa transition, tout en veillant à ne pas glisser sur la pente périlleuse de la symétrisation du cadre prônée par Ferenczi.

Si le/la clinicien/ne doit prôner la fraternité, il importe aussi de privilégier la retenue ainsi que de conserver son énigme. C'est pourquoi nous 
n’avons pas pensé opportun de partager avec David la raison pour laquelle nous serions susceptible de nous tromper concernant son pronom, ainsi que les tenants d'une intersubjectivité ouverte aux éclats de la transparence auraient probablement fait. L'oscillation entre le masculin et le féminin n'était pas simplement due à notre formatage par les normes socioculturelles et/ou métapsychologiques. Elle était infiniment plus complexe et constituait vraisemblablement le contrecoup transférentiel d'une bisexualité psychique et d'une fluidité propres à David, combattues férocement par la mise en place de stratégies de contrôle assez redoutables. S'il a réécrit son passé en s'interdisant d'employer le pronom féminin, ce dernier revenait à l'occasion hanter ses rêves, c'est pourquoi il restait des nuits entières sans sommeil pour éviter que cela ne se reproduise: «Dans le rêve normal, il y a des pensées qui défilent comme dans un film et je n'ai aucune maîtrise; j'ai horreur de ça; je préfère le rêve lucide, celui que je dirige moi-même». Ce fonctionnement laissait évidemment peu de place au questionnement de l'identification transgenre et mettait à l'épreuve notre vocation analytique, à savoir l'exploration des ressorts inconscients qui déterminent la construction subjective. La «magie» transférentielle se révélerait-elle à même de transfigurer cette rigidité?

Nous avons également formulé dans notre esprit une autre question qui nous a inspiré une grande culpabilité: les métapsychologies pathologisantes sur les transidentités résultent-elles d'une simple résistance de la part de l'analyste ou constituent-elles une riposte transférentielle à une résistance vis-à-vis de l'analyse chez les sujets trans'? Existe-t-il uniquement des clinicien/ne/s maltraitant/e/s ou également des patient/e/s agissant/e/s aptes à susciter une connivence sadomasochiste? Rappelons que le contretransfert, bien plus qu'un obstacle de la cure imputable à la subjectivité de l'analyste ou qu'un simple reflet de la pathologie de l'analysant/e, constitue une cocréation des deux parties de la dyade analytique ${ }^{6}$.

David a ouvertement exprimé son appréciation à l'égard de notre attitude honnête et énigmatique à la fois. Notre avertissement concernant la fluctuation entre le prénom masculin et le pronom féminin a semblé induire précisément l'effet décrit par Ferenczi (1933, p. 128): «délier la langue». Ainsi, David s'est-il dorénavant autorisé des plongées plus audacieuses dans son passé en nous révélant des secrets familiaux qui le tourmentaient, tel le soupçon d'une violence incestueuse dont sa mère aurait été victime ou l'assassinat de son grand-père par son épouse: "Lorsqu'on a confiance en son docteur et que celui-ci ne se prend pas pour "Monsieur Je-sais-tout", on 
peut se permettre d'avouer des choses dont on a honte ou des choses qui nous font mal».

Attardons-nous un instant sur l'appellation de «docteur» que David employait à l'occasion pour nous adresser la parole. Faisait-il un amalgame entre le statut du psychiatre et celui du psychologue? Avait-il cherché sur Internet notre profil professionnel mentionnant notre doctorat en sciences humaines? Dans un premier temps, nous avons pensé que cette confusion était imputable au métacadre institutionnel qui cultivait un enchevêtrement entre psychiatres, psychanalystes, psychothérapeutes, psychologues, bénévoles et stagiaires, faute d'une explicitation du statut et du rôle de chacun. Par ailleurs, le fait que nous recevions David dans le bureau du psychiatre qu'il continuait à consulter ponctuellement a dû favoriser notre identification à la fonction de ce dernier. Enfin, l'«omniprésence» du divan dans la pièce a sans doute alimenté la confusion puisque, comme l'a argumenté Roussillon (2005), même lorsque le travail analytique se déroule en face-àface, ce symbole princeps du métier d'analyste adresse au patient assis sur le fauteuil des «messages énigmatiques» imprégnés de significations fantasmatiques inconscientes.

Toutes ces réflexions ont suscité en nous une hostilité à l'égard de la structure et provoqué chez nous un tel vertige que nous ne savions plus qui nous étions, ce que nous faisions, ni comment nous positionner envers David. Cet ébranlement subjectif a commencé à s'atténuer lorsque nous avons compris, dans le cadre de l'intervision, que les problématiques identitaires des patient/e/s se reflétaient probablement dans la dynamique de l'équipe soignante, comme cela peut se produire dans d'autres cas de figure. La conflictualité des sujets que nous écoutions s'était sans doute externalisée et circulait dans «l'espace psychique élargi» de l'institution, pour emprunter l'expression de Philippe Jeammet (1980). Cette prise de conscience, ainsi que le travail d'élaboration que nous avions entrepris dans le cadre d'une supervision collective dédiée aux pratiques cliniques au sein de l'institution, nous a aidé à rétablir progressivement notre cadre interne, à redéfinir les repères spatiotemporels du cadre externe et à redéployer une écoute moins parasitée par notre contretransfert.

\section{La violence des normes de genre}

La plus grande épreuve que David nous a fait endurer était sans doute sa curiosité excessive concernant notre vie privée, qui avait pris une forme paroxystique après qu'il nous avait croisé et filmé lors d'une conférence. 
Pendant plusieurs séances, il nous a répété que l'étude de cette vidéo l'avait persuadé de notre homosexualité. Quelques séances plus tard, il nous a dit soupçonner que nous étions un homme trans', avant de conclure dans un temps ultérieur que nous étions un «métrosexuel» que la dynamique de la séance nous a incité à entendre comme «maître sexuel». Si les projections sur le/la clinicien/ne sont bien évidemment attendues et se prêtent à l'élaboration, David s'est mis par ailleurs à nous espionner et à nous adresser des invitations sur les réseaux professionnels. Il s'est même «magiquement» retrouvé à trois reprises dans le même wagon de métro que nous. Les séances étaient dorénavant saturées de spéculations sur notre sexualité.

Notre refus de satisfaire sa «volonté de savoir» était évidemment dicté par notre désir de rester neutre et de lui accorder la possibilité de réfléchir sur ses identifications projetées ${ }^{7}$. Lisons ce que Denis écrit à ce sujet:

Il faut considérer que la neutralité de l'analyste s'étend à son rôle de support de projection: des caractéristiques trop saillantes peuvent en effet obérer la projection de tout un registre de relations, gauchir les images projetées ou en privilégier trop certaines. Un analyste qui affiche ses préférences de genre, dans un sens ou dans un autre, limite les possibilités d'expression de son patient et est vécu, malgré lui, comme prescripteur d'une attitude. (Denis, 2013, p. 765)

À trop vouloir sauvegarder cette abstention d'attitudes prescriptives de genre, nous avons paradoxalement perverti notre neutralité. Les projections sur nous et les sollicitations sexuelles étaient tellement fréquentes et massives que nous en sommes venu à altérer notre comportement habituel. Des commentaires de type «Vous avez l'air trop maigre (entendre: castré) lorsque vous vous rasez»; «Puisque vous ne dites pas si vous êtes célibataire, je ne viendrai pas la prochaine fois»; «Dites-moi qui vous êtes, ça va me rassurer»; "Je sais pourquoi vous avez choisi de vous occuper des trans'» nous rendaient perplexe, confus ou agacé. Bien plus, lorsqu'il nous parlait du membre viril qu'il rêvait d'avoir, il scrutait les plis de notre pantalon au niveau de l'entrejambe, tandis que, à d'autres moments, il nous fixait de façon analogue, stimulé par une luxure nullement voilée. Une fois n'est pas coutume, en tant que clinicien, notre corps comptait autant, voire davantage que notre parole, ce qui nous était parfois pénible. Nous nous sommes donc surpris à endosser des tenues sombres, à «oublier» de nous raser pour éviter de perdre nos "attributs phalliques", ou à adopter des postures de 
type jambes largement ouvertes et fermement posées sur le sol, pour correspondre aux traits du mâle radicalement stéréotypé que David projetait sur nous.

Nous avons fini par comprendre que nous reproduisions défensivement l'identification aux normes de genre rigides que notre patient avait incorporées au prix d'une violence qu'il nous faisait vivre à son tour par ses divers commentaires sexistes et l'incitation forcée à l'autodéfinition. David était bien agissant et nous lui en voulions. Il nous bombardait sans cesse de messages prescriptifs et nous en étions saturé. Il en devenait maltraitant et nous en avions assez. Cette prise de conscience n'a pas cependant automatiquement écarté notre embarras, puisque, comme le précise J.-B. Pontalis (1975, p. 75), reconnaître le contretransfert n'implique pas forcément que l'on s'en débarrasse, "car la résistance au contretransfert est tout autant présente dans le: “je sais où j'en suis”, qu'elle le serait chez l'analysé qualifiant par exemple son transfert de "maternel" pour contenir, tenir sous le joug du mot et de l'image, des émois inqualifiables ». Cet avertissement suggère la nécessité d'appliquer au contretransfert ce que Freud (1914) préconisa en pensant aux analysants: la perlaboration de leurs résistances. En conséquence, il importe pour le thérapeute d'accomplir de manière analogue au patient une traversée de ses fantasmes et de travailler perpétuellement le décentrage de ses préjugés cliniques, théoriques, socioculturels et «trans-sexuels», au sens que Nahon donne à ce terme:

La subversion des identités en vigueur dans l'inconscient, leur combinaison extrême, cet ordonnancement très singulier du masculin et du féminin qui transparaît dans les formations de rêve. La trans-sexualité déloge, distord et diffracte le sexe et la fixité de ses représentations, au premier rang desquels, évidemment, l'idée d'une «sexualité de l'homme», d'une «sexualité de la femme», a fortiori, l'hétérosexualité et l'homosexualité. (2006, p. 23)

\section{La tyrannie du politiquement correct}

Nous terminerons notre narration par un épisode de la cure de David qui illustre de manière emblématique la difficulté à conserver une écoute proprement analytique dans le contexte actuel des transidentités. Si la mise à l'épreuve de notre neutralité était liée jusqu'à présent aux problématiques intrapsychiques du patient ainsi qu'à nos propres préjugés et réactions inconscientes, à ces facteurs est venue s'ajouter une complexité d'ordre 
politique. Au tout début de sa thérapie, David s'est remémoré une scène traumatique de son enfance au cours de laquelle sa mère aurait battu son père sous ses yeux avec une violence inouïe, tout en expliquant à sa «fille»: «voilà comment une femme doit se comporter envers les hommes». Au moment précis de l'émergence de ce souvenir, David a interrompu son récit afin de nous demander si nous pouvions intervenir pour que son médecin augmente ses doses de testostérone. Sa requête était à peine voilée: ne désirait-il pas que nous l'assistions pour qu'il se différencie le plus possible de cette mère terrifiante?

Deux ans plus tard, lorsque David est revenu sur l'incident survenu au cours de son enfance et que la dynamique de la séance nous a incité à penser que nous pouvions lui proposer un lien entre ce souvenir et la prise d'hormones mâles, il nous a accusé avec véhémence d'invalider sa démarche de transition et de remettre en question l'authenticité de son identité et menacé de nous dénoncer aux associations trans'. S'il est revenu pour la séance suivante et a pu se ressaisir de notre interprétation en élaborant celle-ci de manière très riche, force nous est d'avouer que nous demeurons aujourd'hui encore troublé par sa réaction initiale et hanté par une espèce d'«injonction paradoxale»: d'une part, nous serions missionné pour conduire une psychothérapie et, d'autre part, nous serions privé du droit de questionner, voire du droit de penser.

Nous émettrons à cet égard l'interrogation suivante: dans le contexte d'un discours politiquement correct et d'un militantisme qui a transformé le stigmate des transidentités en "puissance d'agir» (agency) et source de création (Califia, 2003; Preciado, 2008; Butler 2009; Saketopoulou, 2014; Gozlan 2018), le/la clinicien/ne analytique a-t-il/elle le droit d'interroger les identités trans' sans se voir taxer de normativité? Est-il loisible aujourd'hui de questionner les ressorts inconscients qui surdéterminent les identifications transgenres à l'instar de toute identification typique ou atypique? Éluder ce questionnement au nom de la «naturalité» de ce choix identitaire, ainsi que le revendique souvent le discours progressiste, ne reviendrait-il pas à tomber dans le même piège qu'une certaine norme majoritaire encline à s'interdire toute interrogation de l'identité hétérosexuelle cis-genre?

Dans un article intitulé "L'hétérosexualité: une formation de compromis» (Heterosexuality as a Compromise Formation), la psychanalyste et féministe américaine Nancy Chodorow (1992) a montré comment la «naturalisation» de l'hétérosexualité en tant qu'orientation dominante a longtemps entravé sa remise en question et a conduit à la scotomisation de la 
conflictualité intrinsèque à la subjectivité humaine. L'idée que nous aimerions souligner ici, c'est que toute revendication essentialisante et toute forme de police du discours risquent de mener à un aveuglement et à une coercition de la pensée. Le défi analytique consiste, nous semble-t-il, à trouver un équilibre entre une mise en sens des processus de subjectivation (trans', cis, bi, homo, hétéro, etc.) et une mise à l'écart de tout type de normativité hétérocentrée ou queercentrée. Rappelons avec Jacques André (2018) que l'inconscient est par définition a-normatif et politiquement incorrect.

\section{Réflexions conclusives}

Aujourd'hui, nous sommes enclin à penser que la direction de la cure de David était imprégnée d'un certain manichéisme, opposant ce qui serait «bien» à ce qui serait «mal» au détriment d'une prise en considération exhaustive de l'ambivalence et de l'extrême complexité des conjectures cliniques des transidentités. La présente contribution porte sans doute les traces de notre difficulté à nous dégager pleinement de notre contretransfert, malgré nos efforts soutenus de per-laboration (Freud, 1914) ou, selon une autre traduction du terme freudien Durcharbeitung qui fait davantage écho aux problématiques abordées ici, de trans-laboration.

En guise de conclusion, nous préciserons que nous avons entrepris une supervision du cas de David avec un analyste hiérarchiquement et générationnellement supérieur. En dépit de son intérêt, ce travail a reproduit méta-transférentiellement les injonctions écrasantes que le patient en question aurait subies dans son enfance et a induit chez nous les effets de ce que Ferenczi (1932) a appelé l' «intro-pression ${ }^{8} »$, à savoir l'introjection du surmoi d'un père superviseur et la répression de nos fonctions moïques. À la suite de cette expérience qui mériterait que nous lui consacrions un développement à part entière, nous avons opté pour une intervision avec quelques pairs. Cette autre dimension de métaréflexion clinique, qui a su mobiliser chez nous des identifications horizontales plus plastiques par rapport à certaines identifications verticales rigidifiées, nous a permis de faire varier notre écoute plus librement et d'intégrer nos erreurs sans les reproduire défensivement dans l'espace de la cure. Par ailleurs, l'écriture que nous avions entreprise sur ce cas a lancé un mouvement parallèle d'auto-analyse et d'auto-théorisation qui a favorisé notre affranchissement de l'emprise de certains pré-cadres conceptuels ou signifiants-maîtres idéalisés. En définitive, pour paraphraser l'aphorisme de Winnicott «un bébé tout seul, ça n'existe pas», un/e clinicien/ne tout/e seul/e, cela n'existe pas non plus. C'est 
pourquoi nous avons besoin de nouvelles tranches d'analyse, de supervisions, d'intervisions, de lectures disciplinaires et transdisciplinaires, ainsi que d'auto-supervisions par l'écriture, telle la rédaction du présent article.

\author{
Nicolas Evzonas \\ nicolas.evzonas@gmail.com
}

\title{
Notes
}

1. Dans son dernier article sur les transidentités, Lemma (2018) discute ce sujet de manière intéressante.

2. Cf. la discussion par Gozlan (2018), qui, malgré son positionnement progressiste en faveur des identités trans', récuse les termes «transgénérisme» et «transidentités» au motif que ces derniers font fi de la sexualité - à vrai dire le sexuel infantile - consubstantielle à l'identification et au genre. Il se réapproprie ainsi le vocable médico-psychiatrique «transsexualisme», lourdement chargé de connotations pathologiques, dans une perspective de retournement du stigmate, à l'instar de l'insulte "queer» réinvestie et resignifiée au sein de la communauté LGBTQIA.

3. Voir la discussion de ces positions analytiques par Espineira (2015), Ayouch (2015) et Hansbury (2017).

4. Voir la critique de ces analystes par Ayouch (2015).

5. On lira avec intérêt l'article récent de Saketopoulou et Pellegrini (2019) dédié aux défis contretransférentiels relatifs à l'utilisation des pronoms dans le cadre de la cure analytique des patient/e/s non-binaires.

6. Voir les approches contemporaines du contretransfert dans l'ouvrage collectif dirigé par Oelsner (2013).

7. Nous reprenons dans ce segment les réflexions que nous avons développées dans notre article «La violence "pousse-à-l'homme": approche clinique d'une subjectivation transmasculine» (Evzonas, 2019, p. 29-31).

8. Voir l'analyse de cette notion par Martín Cabré (2011).

\section{Références}

André, J. (2018). L'inconscient est politiquement incorrect. Paris: Stock.

Aulagnier, P. (1984). L'apprenti-historien et le maître-sorcier. Paris: Presses universitaires de France.

Ayouch, T. (2015). Psychanalyse et transidentités: hétérotopies. L'évolution psychiatrique, 80, 303-316.

Barkai, A. R. (2017). Troubling Gender or Engendering Trouble? The Problem with Gender Dysphoria in Psychoanalysis. The Psychoanalytic Review, 104(1), 1-32.

Butler, J. (2009). Le transgenre et les «attitudes de révolte». Dans M. David-Ménard (dir.), Sexualités, genres et mélancolie (p. 13-36). Paris: Campagne première.

Califia, P. (2003). Le mouvement transgenre. Paris: EPEL.

Castel, P. H. (2003). L'impensable métamorphose. Paris: Gallimard.

Chiland, C. (2011). Changer de sexe: Illusion ou réalité. Paris: Odile Jacob.

Chodorow, N. J. (1992). Heterosexuality as a compromise formation: Reflections on the psychoanalytic theory of sexual development. Psychoanalysis and Contemporary Thought, 15(3), 267-304. 
Davidson, A. I. (1987). Sex and the emergence of sexuality. Critical Inquiry, 14(1), 16-48.

Denis, P. (2013). Transfert et réaction transférentielle de genre. Revue française de psychanalyse, 77 (1), 762-769.

Didi-Huberman, G. (1999). Ce que nous voyons, ce qui nous regarde. Paris: Éditions de Minuit. Espineira, K. (2015). Transidentités: Ordre et panique de genre. Paris: L'Harmattan.

Evzonas, N. (2019). La violence "pousse-à-l'homme»: approche clinique d'une subjectivation transmasculine. Psychologie clinique et projective, 25 (1), 11-33.

Fassin, E. (2003). L'inversion de la question homosexuelle. Revue française de psychanalyse, 67 (1), 263-284.

Ferenczi, S. (1928). Élasticité de la technique psychanalytique. Dans Euvres complètes, vol. IV (p. 53-65). Paris: Payot, 1982.

Ferenczi, S. (1932). Le traumatisme psychique. Dans Cuvres complètes, vol. IV (p. 315-316). Paris: Payot, 1982.

Ferenczi, S. (1933). Confusion de langue entre les adultes et l'enfant. Dans Euvres complètes, vol. IV (p. 125-138). Paris: Payot, 1982.

Freud, S. (1911). Remarques psychanalytiques sur l'autobiographie d'un cas de paranoïa. Dans Cinq psychanalyses. Paris: Presses universitaires de France, 1977.

Freud, S. (1914). Remémoration répétition et perlaboration. Dans De la technique psychanalytique. Paris: Presses universitaires de France, 1981.

Gabbard, G. (2000). Disguise or Consent. Problems and Recommendations Concerning the Publication and Presentation of Clinical Material. The International Journal of Psychoanalysis, 81(6), 1071-1086.

Gozlan, O. (2008). The Accident of Gender. The Psychoanalytic Review, 95(4), 541-570.

Gozlan, O. (2018). Introduction. Dans O. Gozlan (dir.), Current Critical Debates in the Field of Transsexual Studies. Londres/New York: Routledge.

Hansbury, G. (2011). King Kong and Goldilocks: Imagining transmasculinities through the trans-trans dyad. Psychoanalytic Dialogues, 21(2), 210-220.

Hansbury, G. (2017). Unthinkable Anxieties: Reading Transphobic Countertransferences in a Century of Psychoanalytic Writing. Transgender Studies Quarterly, 3-4(4), 384-404.

Jeammet, P. (1980). Réalité interne et réalité externe dans l'adolescence. Revue française de psychanalyse, 44 (3), 481-521.

Kristeva, K. (2003). Chroniques du temps sensible. Paris: Éditions de l'Aube.

Lacan, J. (1969-1970). L'envers de la psychanalyse. Paris: Seuil, 1991.

Laplanche, J. (1987). Nouveaux fondements pour la psychanalyse. Paris: Presses universitaires de France.

Laplanche, J. (2007). Sexual: La sexualité élargie au sens freudien (2000-2006). Paris: Presses universitaires de France.

Laqueur, T. (1992). La fabrique du sexe: Essai sur le sexe et le genre en Occident. Paris: Gallimard. Laufer, L. (2010). La fabrique du corps sexué. Recherches en psychanalyse, 10 (2), 231-241.

Lemma, A. (2018). Trans-itory Identities: Some Reflections on Transgender Identities. The International Journal of Psychoanalysis, 99(6), 1089-1106.

Lothstein, M. L. (1979). Group Therapy with Gender Dysphoric Patients. American Journal of Psychotherapy, 33, 67-81.

Martín Cabré, L. J. (2011). From Introjection to Intropression: Evolution of a Theoretical Concept and its Consequences for Psychoanalytic Technique. The American Journal of Psychoanalysis, 71(4), 321-328.

Nahon, C. (2006). La trans-sexualité ou l'en-dehors des formes. Cliniques méditerranéennes, 74, 5-26. 
Neyraut, M. (1974). Le transfert: Étude psychanalytique. Paris: Presses universitaires de France.

Oelsner, R. (dir.) (2013). Transference and Countertransference Today. Londres: Routledge.

Pontalis, J. B. (1975). À partir du contretransfert: le mort et le vif entrelacés. Nouvelle revue de psychanalyse, 12, 73-88.

Preciado, B. P. (2008). Testo Junkie: Sexe, drogue et biopolitique. Paris: Grasset.

Roussillon, R. (1991). Paradoxes et situations limites de la psychanalyse. Paris: Presses universitaires de France.

Roussillon, R. (2005). La «conversation» psychanalytique: un divan en latence. Revue française de psychanalyse, 69 (2), 365-381.

Saketopoulou, A. (2014). Mourning the Body as Bedrock: Developmental Considerations in Treating Transsexual Patients Analytically. Journal of the American Psychoanalytic Association, 62(5), 773-805.

Saketopoulou, A. et Pellegrini, A. (2019). On taking sides: they/them pronouns, gender and the psychoanalyst. Psychoanalysis today, 8 ( "Sexualities and Diversity»). Repéré à http:// www.psychoanalysis.today/fr-FR/PT-Articles/Pellegrini167541/On-taking-sides-theythem-pronouns,-gender-and-the.aspx

Searles, H. (1959). The effort to drive the other person crazy - An element in the aetiology and psychotherapy in schizophrenia. British Journal of Medical Psychology, 23(1), 1-18. 